\title{
Effects of Shoes and a Prefabricated Medial Arch Support on Medial Gastrocnemius and Tibialis Anterior Activity while doing Leg Press Exercise in Normal Feet Athletes
}

\author{
Maryam Sheikhi \\ Department of Physiotherapy, School of Rehabilitation Sciences, Shiraz University of Medical science. Shiraz, Iran \\ E-mail: m.sheykhi.m@gmail.com \\ Soraya Piroozi (Corresponding author) \\ Department of Physiotherapy, School of Rehabilitation Sciences, Shiraz University of Medical science. Shiraz, Iran \\ E-mail: piroozis@sums.ac.ir \\ Mohsen Mirzaie Khoshalani \\ Department of Physiotherapy, School of Rehabilitation Sciences, Shiraz University of Medical science. Shiraz, Iran \\ E-mail: mirmowj@gmail.com \\ Tahere Motiallah \\ Department of Physiotherapy, School of Rehabilitation Sciences, Shiraz University of Medical science. Shiraz, Iran
}

Seyed Hasan Zolanvar

School of mechanical engineering, Shiraz University, Shiraz 71936-16548 Iran

E-mail: s.hasan.zolanvar@gmail.com

Received: 21-01- 2017

doi:10.7575/aiac.ijkss.v.5n.2p.31
Accepted: 10-04- 2017

Published: 30-04- 2017

\begin{abstract}
Background: Nowadays, different types of exercise machines are being used in the field of athletic training, recreation, post-injury and post-operation rehabilitation. Leg press is a commonly-used one that retrains muscles and simulates natural functional activities. In this activity, feet are in contact with a footrest to exert muscular forces. In addition, the footrest inserts reactive forces to feet and from the feet load would transfer to structures that are more proximal. Any misalignment in foot structure may interfere its function. Objective: The aim of this study was to assess the effect of shoes and using a prefabricated medial arch support on the activity of Tibialis anterior and medial gastrocnemius muscles while doing leg press exercise in normal feet subjects. Method: 14 men with normal Medial Longitudinal Arch and normal Body Mass Index aged between 18-35 years old, with at least 6 months experience of doing leg press volunteered to participate in this study. Medial gastrocnemius and Tibialis anterior activity were measured by surface electromyography while doing leg press with $70 \%$ of subjects 1 Repetition Maximum. To increase accuracy, motion was divided into knee flexion and knee extension phases. Peak Amplitude, Time to Peak Amplitude and Root Mean Square variables were used for analysis. Wilcoxon nonparametric test was used to compare the results. Results: No statistically significant difference was found in the electromyographic parameters of Medial gastrocnemius nor Tibialis anterior in any phases of motion, except for an increase in Tibialis anterior time to peak amplitude in shod condition compared with barefoot in knee extension phase of motion (p-value $=0.008$ ) and Tibialis anterior RMS in knee flexion phase in orthotic condition compared to shod ( $\mathrm{p}$-value=0.03). Conclusion: It seems that in high loads shoes or medial arch supports cannot change electromyographic parameters in Medial gastrocnemius nor Tibialis anterior in any phase of motion while working with leg press device.
\end{abstract}

Keywords: Foot Structure, Surface electromyography, Foot Arch Support.

\section{Introduction}

Foot is one of the most important parts of the body that bears and distributes weight in close kinetic chain activities. This ability occurs through the complexity of its joints and its well-known Medial Longitudinal Arch (MLA) that has the ability to compress and recoil in response to different contact surfaces (Kelly, Cresswell, Racinais, Whiteley, \& Lichtwark, 2014; Letafatkar, Zandi, Khodayi, \& Vashmesara, 2013). In fact, it is the only part of body that is in contact with supporting surfaces in different activities and plays a main role in load transportation to most proximal parts of body. Any misalignment in foot biomechanics may impose abnormal stresses to lower extremity that can lead to injury (Murphy, Connolly, \& Beynnon, 2003; Qaqish \& McLean, 2010a). Health professionals frequently encourage people to 
exercise regularly because of its positive effects on well-being. When suggesting a subject to do exercise for wellbeing, body may be placed in challenging positions (Keller et al., 1996). The more vigorous the activities, the more concern we will have about loud on lower limbs and even spinal column. In exercises where feet are in contact with supporting surface, the importance of their normal structure and function becomes vital in dealing with stress (Fu et al., 2016; Tenforde, Yin, \& Hunt, 2016). Nowadays, people choose to exercise with equipment. This has become more popular because exercise intensity and other features are more controllable and it is believed that the movements are done in a safer situation. Leg press is one of the common exercises that increase lower extremity muscular strength prescribed in early rehabilitation programs to retrain lower extremity muscles and simulate natural functional activities (D'Lima, Patil, Steklov, \& Colwell Jr, 2011; Gibson \& Habing, 1994; Wawrzyniak, Tracy, Catizone, \& Storrow, 1996). In leg press, feet are in contact with a footrest to insert muscular forces. In addition, the footrest inserts reactive forces to the feet and from feet to more proximal structures. In professional athletes, loads transferred via feet is occasionally much more than the body weight and may interfere with functions of intrinsic and extrinsic muscles attached to bony structures. Some are known as dynamic supports of the MLA and others are further muscles such as back and neck. The aim of this study was to examine the effect of shoes and a semi-rigid prefabricated medial arch support on Medial gastrocnemius and Tibialis anterior muscular activity in athletes with normal medial longitudinal arch while working with leg press device. These two muscles are considered to control foot motion and support medial longitudinal arch dynamically. Muscle activity was analyzed in the entire knee flexion and knee extension motion range. Exercise intensity was about $70 \%$ for each subject 1RM in all three foot conditions (R. F. Escamilla et al., 2001). Leg press load has been assumed to work as an input signal to change muscular activity subsequent to the changes in the alignment of feet bony structures. The effect of using shoes and a prefabricated medial arch support on Medial Gastrocnemius and Tibialis Anterior Activity was examined while doing leg press exercise in normal feet subjects (Nigg, 2001). Previous studies have focused on changes of muscular activity as a result of using shoes or orthoses during walking and running or while doing squat exercise (Larson, 2013; Murley \& Bird, 2006; Tomaro \& Burdett, 1993). Therefore, the objective of this study was to investigate the effect of wearing shoes or using a prefabricated medial arch support on muscular activity of Tibialis anterior and medial gastrocnemius muscles in normal feet athletes while doing leg press.

\section{Method}

\subsection{Participants of the study}

The participants were selected among volunteer male athletes aged 18 to 35 years old with at least six months of experience doing leg press exercise. Navicular drop test was performed to assess the medial longitudinal arch and subsequently 14 subjects with normal medial arch (between 6-9 mm) (Sporndly-Nees, Dasberg, Nielsen, Boesen, \& Langberg, 2011) and normal body mass index (BMI level 18.5-<25) (Stevens, 2014) were recruited to participate in this study.

\subsection{Test procedure}

The test procedure was submitted to and approved by Vice Chancellor for Research Affairs, Shiraz University of Medical Sciences. After explaining the experimental procedure, written informed consent form was obtained from the subjects. Exclusion criteria were any biomechanical dysfunction, which made the subjects unable to do the tests, current or previous foot pain, any history of lower extremity surgery, injury to the lower extremity in the last 6 months and pain during walking (Ferber \& Benson, 2011; Franettovich, Murley, David, \& Bird, 2012)

Test procedure and skin preparation was explained to all subjects. The test was performed in 2 different days with oneday interval to lessen the fatigue effect. On the first day, demographic information was obtained; then, a subject 1repetition maximum (1RM) was measured. To assess $1 \mathrm{RM}$, the subjects had to jog 3-5 minutes as warm up. After sitting on leg press load was gradually increased during 4-5 sets with 5 min rest between each set or until the subject did not feel tired. On the second day, after electrode placement Maximal Voluntary Isometric Contraction (MVIC) was measured 3 times in a 6-second period, with 5 min rest between each repetition and with the vocal encouragement of the examiner. The middle 2 seconds of each 6-second contraction was recorded and the average between the 3 trials was used as MVIC(C. Eleanor 2010) Tibialis anterior and Medial gastrocnemius electromyographic activity was recorded by circular surface electrodes (ME 6000 device, USA) (Franettovich et al., 2012; Qaqish \& McLean, 2010a). Subjects were applying maximal effort for each muscle according to positions and methods used in manual muscle testing (R. F. Escamilla, Francisco, Kayes, Speer, \& Moorman 3rd, 2002; Murley \& Bird, 2006). For Tibialis anterior surface electrodes were placed on the $1 / 5$ proximal of the distance between tibial tuberosity and the midline between medial and lateral malleoli's. Medial gastrocnemius electrodes were placed on 1/4 of proximal of the distance between medial popliteal fossa and calcaneal tubercle. Reflective markers were placed on bony landmarks according to the device protocol and knee kinematics were quantified by the use of six-camera 3-D motion analysis system $(100 \mathrm{~Hz}$, Qualisys Track Manager, Sweden) (Chang, Hung, Wu, Chiu, \& Hsu, 2010).

According to the maximal knee flexion angle, the motion was divided into 2 phases: down or knee flexion phase (from the beginning to maximal knee flexion) and up or knee extension phase (from maximal knee flexion to full knee extension)(R. F. Escamilla et al., 2001). All EMG data was normalized to subjects MVIC. Each subject did the test at his convenient speed and finally EMG data was divided to the motion duration to omit the speed effect. At first, the raw EMG was high-pass filtered using a zero-lag fourth-order Butterworth filter $(30 \mathrm{HZ})$ to remove movement artifact; then full waves were rectified and filtered using a Butterworth low-pass filter with a 6HZ low-pass cut off frequency (Lloyd $\&$ Besier, 2003). After normalizing data peak amplitude, time to peak amplitude, and Root Mean Square variables were 
used to compare results. Time to peak amplitude was calculated as a percentage of total phase time and in each phase of motion peak amplitude was the maximal muscle activity.

(a)

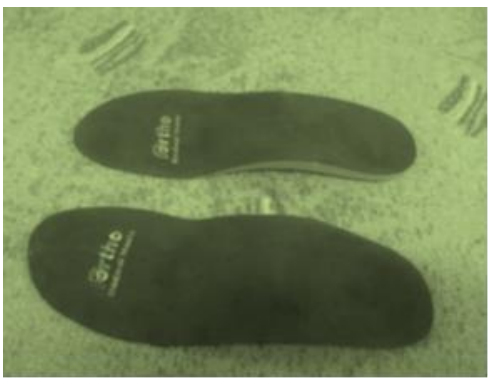

(b)

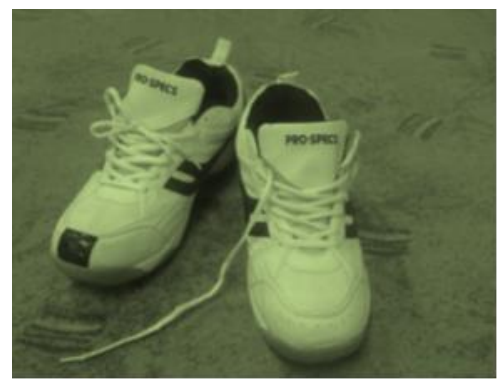

Figure 1. Prefabricated medial arch insole (a) and shoes (b) used in this study.

A 45-degree leg press equipment (PANATTA Co. 45 ) was used. The distance between subjects feet on the footrest was the same as distance between their anterior superior iliac spines (R. F. Escamilla et al., 2001) in all 3 test conditions. Data recording began as the motion started (Bezerra et al., 2013). A prefabricated semi-rigid medial arch support with 2 degrees of medial wedge used in this study was made by Kinsiofit Company in Iran and the shoes were commonly known as squat shoes by athletes (Figure 1).

\subsection{Statistical analysis}

Statistical analysis was done by the use of SPSS 18 software. Since the data were not normally distributed,-Wilcoxon nonparametric test was utilized to compare results. This nonparametric test solves any problem about standard deviations near or greater than the means as well as data normality. P-value $=0.05$ was considered as the significant level.

\section{Results}

The results of current study demonstrated that wearing shoes or using a prefabricated medial arch support could not change Medial gastrocnemius and Tibialis anterior muscular activity in normal feet subjects while doing leg press.

There were no statistically significant differences between three test conditions in Medial gastrocnemius and Tibialis anterior neither in knee flexion nor knee extension phases (Table 1). Time to peak increased in both studied muscles during knee flexion and knee extension phases in shod and prefabricated medial insole conditions in comparison with barefoot and in prefabricated medial insole condition compared to shoes alone. However, these increases were not statistically significant in any of situations, except for Tibialis anterior in knee extension phase in comparison with shod $(48 \pm 35)$ and barefoot $(26 \pm 38)$ conditions (p-value $=0.008)$ and Tibialis anterior RMS in knee flexion phase in orthotic condition $(16 \pm 13)$ compared to shod $(14 \pm 11)(p$-value=0.03). $($ Table 2$)$.

Table 1. Normalized \%MVIC mean \pm SD EMG activity for Medial gastrocnemius and Tibialis anterior muscles in knee flexion and knee extension phases of motion in barefoot, shod and orthotic conditions

\begin{tabular}{|c|c|c|c|c|c|c|c|}
\hline & \multirow[t]{2}{*}{ Foot condition } & \multicolumn{3}{|c|}{$\begin{array}{l}\text { Knee flexion } \\
(\text { mean } \pm \text { SD) }\end{array}$} & & \multicolumn{2}{|c|}{$\begin{array}{l}\text { Knee extension } \\
(\text { mean } \pm \mathrm{SD})\end{array}$} \\
\hline & & $\mathrm{PA}(\mu v)$ & TTP(msec) & $\operatorname{RMS}(\mu \nu)$ & $\mathrm{PA}(\mu v)$ & $\mathrm{TTP}(\mathrm{msec})$ & $\operatorname{RMS}(\mu \nu)$ \\
\hline & Barefoot & $29 \pm 19$ & $66 \pm 41$ & $15 \pm 9$ & $30 \pm 17$ & $47 \pm 36$ & $17 \pm 9$ \\
\hline \multirow[t]{3}{*}{ MG } & Shod & $30 \pm 21$ & $80 \pm 32$ & $15 \pm 10$ & $29 \pm 18$ & $51 \pm 31$ & $18 \pm 10$ \\
\hline & Orthotic & $30 \pm 26$ & $91 \pm 19$ & $15 \pm 13$ & $27 \pm 20$ & $58 \pm 40$ & $16 \pm 12$ \\
\hline & Barefoot & $36 \pm 30$ & $76 \pm 32$ & $17 \pm 14$ & $26 \pm 29$ & $26 \pm 38$ & $14 \pm 15$ \\
\hline \multirow[t]{2}{*}{ TA } & Shod & $29 \pm 23$ & $89 \pm 16$ & $14 \pm 11$ & $25 \pm 25$ & $48 \pm 35$ & $14 \pm 15$ \\
\hline & Orthotics & $33 \pm 29$ & $70 \pm 32$ & $16 \pm 13$ & $27 \pm 26$ & $46 \pm 44$ & $15 \pm 15$ \\
\hline
\end{tabular}

*MVIC: Maximal Voluntary Isometric Contraction; PA: Peak Amplitude, TTP: Time to Peak amplitude, RMS: Root Mean Square, TA: Tibialis Anterior muscle, MG: Medial Gastrocnemius. 
In knee flexion phase, negligible rises of mean \pm SD in RMS and PA between different conditions were seen in MG. Mean \pm SD TTP had more increases between various foot conditions but none were statistically significant. Also in this phase, the increased RMS in orthotic condition compared to shod was statistically significant. In knee extension phase, neither slight falls in MG peak amplitude nor increases of MG time to peak were significant. In this phase, MG root mean square showed a fluctuating trend in diverse conditions, which were not significant at all. In knee extension phase, RMS and PA remained almost unchanged in all three conditions. TTP increased significantly in shod condition compared to barefoot.

Table 2. Correlation-coefficient between variables in knee flexion and knee extension phases of motion in different foot conditions

\begin{tabular}{llllllll}
\hline & Foot condition & \multicolumn{3}{c}{$\begin{array}{c}\text { Knee flexion } \\
\text { (P-value) }\end{array}$} & & \multicolumn{3}{c}{$\begin{array}{c}\text { Knee extension } \\
\text { (P-value) }\end{array}$} \\
& & PA( $\mu v)$ & TTP(msec) & RMS $(\mu v)$ & PA $(\mu v)$ & TTP(msec) & RMS $(\mu v)$ \\
\multirow{2}{*}{ MG } & Barefoot-Shod & 0.778 & 0.424 & 0.0925 & 0.433 & 0.701 & 0.826 \\
& Shod-Orthotic & 0.826 & 0.139 & 0.778 & 0.730 & 0.778 & 0.272 \\
& Barefoot- & 0.875 & 0.050 & 0.925 & 0.300 & 0.158 & 0.875 \\
& Orthotic & & & & & & \\
& Barefoot-Shod & 0.177 & 0.576 & 0.300 & 0.594 & 0.008 & 0.551 \\
TA & Shod-Orthotic & 0.140 & 0.099 & 0.030 & 0.826 & 0.695 & 0.925 \\
& Barefoot- & 0.730 & 0.249 & 0.975 & 0.683 & 0.074 & 0.551 \\
& Orthotic & & & & & & \\
\hline
\end{tabular}

*PA: Peak Amplitude, TTP: Time to Peak amplitude, RMS: Root Mean Square, TA: Tibialis Anterior muscle, MG: Medial Gastrocnemius.

Some of the comparisons were close to the significant level, but statistically, just TA root mean square in knee flexion phase in orthotic condition increased compared to shod and in knee extension phase its TTP increased in shod compared to barefoot condition.

\section{Discussion}

During foot loading conditions, like contact phase of walking, the arch is under pressure to flatten; thus, tension is created along the plantar fascia to maintain foot structure under load (Franklin, Grey, Heneghan, Bowen, \& Li, 2015). In the other side, the neuromuscular system generate responses based on afferent sensory information produced by mechanical loads placed on the foot (Sacco, Akashi, \& Hennig, 2010). There are 104 cutaneous mechanoreceptors located in the foot sole. Receptor distribution is primarily where the foot is in contact with the ground, and no background activity is found when the foot is unloaded (Kennedy \& Inglis, 2002). In addition, there are more fast adapting units than slow suggesting a high dynamic sensitivity. Any increase in plantar sensory feedback in medial side of foot leads to an immediate decrease of mid foot pronation while walking. In loading phase, with an increase of plantar feedback, midfoot angles become more supinated (Kennedy \& Inglis, 2002; Ritchie, Paterson, Bryant, Bartold, \& Clark, 2011). In leg press with $70 \%$ of the subjects 1RM, the load exerting to foot sometimes becomes much greater than the body weight. The results of the current study suggest that midfoot joints reaction is directly related to the magnitude of stimulations in these mechanoreceptors. It means that stimulation of these mechanoreceptors may cause midfoot supination in leg press. This supination helped the foot to tolerate increased load and resist against more midfoot pronation in our normal feet subjects. Second, due to lack of any previous similar research, we used squat to justify our findings. Similar to leg press, squat has two phases of motion: knee flexion and extension. Given the fact that Gastrocnemius is a biarticular muscle, its length may not significantly change during squat. The reason is that in knee flexion phase, it shortens on knee and lengthens on ankle joint and in knee extension phase vice versa (lengthens on knee and shortens on ankle)(R. F. Escamilla et al., 1998). During squat in knee extension phase, it eccentrically contracts to decrease the rate of ankle dorsiflexion and concentrically contracts to help ankle plantarflexion. With respect to force-length relationship in skeletal muscles, constant muscle length helps it to generate better force and control motion alongside other muscles. Our results can be in direction of previous studies which acclaim that medial gastrocnemius has just a moderate activity while doing leg press(R. F. Escamilla, 2001). This moderate activity shows that regardless of foot type or foot wear, gastrocnemius plays its main role in controlling the knee and ankle joints in activities such as squat and leg press. Hence, probable changes in foot structure under high loads or by wearing shoes or orthotics cannot interfere with its function and activity. Results of the present study support the idea that medial gastrocnemius is a motion controller and proves this idea that medial gastrocnemius activity is not completely influenced by foot posture. It is doubtful that medial gastrocnemius can be a rear foot invertor since deviations in rear foot cannot change its activity (Murley, Menz, \& Landorf, 2009; Tomaro \& Burdett, 1993). Tibialis anterior is another muscle which was investigated in this study. It is placed in the medial side of subtalar joint and is responsible for pronation deceleration in this joint (Murley \& Bird, 2006). If the subtalar joint is in a pronated position, the osseous stability of the foot will be disrupted, resulting in excessive foot motion (Tomaro \& Burdett, 1993). Studies have shown no difference in Tibialis anterior activity during the stance phase among 
pes planus, pes cavus and normal feet asymptomatic subjects. Only excessive postures of pronation and supination limit the ability of foot interaction and, thus increases the demand in musculoskeletal structures, which activate to maintain body stability. This can cause abnormal tensile stresses on external foot muscles such as Tibialis anterior and gastrocnemius (Qaqish \& McLean, 2010b). Our results confirmed this finding, showing that shoes and orthosis cannot change Tibialis anterior activity. In leg press, proximal muscles are more involved in generating force. Shoes and orthoses may exert an effect on those muscles. It is thus recommended to perform this test by studying the more proximal muscles like erector spinae, glutei, quadriceps, and hamstrings (Escamilla et al., 1997). Orthoses can decrease maximal hip adduction and knee medial rotation angle. Ankle dorsiflexion angle also decrease as a result of the decrease in hip adduction, and "skeletal realignment" with the use of shoes, insoles and orthoses should be revised (Lack et al., 2014). This study could be in line with the idea that using orthoses can change kinematic in proximal parts in the kinetic chain. Previous neuromuscular experience of subjects and appropriate co-contraction between their muscles might have influenced our findings. Subjects were assessed with just three repetitions for each situation and approximately moderate break between the test conditions. It may be possible that in real conditions when subjects do leg press with a loading range of 10-12 repetitions, the fatigue effect in muscles alters their function during leg press. (de Moura, de Souza Bezerra, da Rosa Orssatto, Sakugawa, \& Diefenthaeler, 2016). Therefore, it is highly recommended that this study be repeated considering the fatigue effect in these muscles. Another probable cause for our results could be the heavy load that was examined in this study. It is possible that the amount of resistance was so high that shoes and orthotics failed to hold against it and no differences was observed as a result of wearing them in any of the conditions. Due to practical constraints, this paper cannot provide a comprehensive review of reaction forces and foot bones osteokinematic motions. Therefore, it is highly recommended to repeat the study assessing reaction forces as well as kinematic parameters. Moreover, one of the most important dynamic supports for medial longitudinal arch is Tibialis posterior muscle, which should be studied by fine wire EMG. We suggest a similar study to be performed on this muscle. We also recommend this study be conducted with less resistance.

\section{Conclusion}

Wearing shoes did not dramatically influence Tibialis anterior and medial gastrocnemius muscles activity nor did medial arch supports in normal feet athletes. Based on our results, fatigue, experience of doing leg press and load are contributing factors that may have affected our findings.

\section{Acknowledgment}

All the authors would like to show their gratitude to Vice Chancellor of Research at Shiraz University of Medical Sciences for their support and all those athletes who participated in this research. The authors would also like to thank the Research Consultation Center (RCC) of Shiraz University of Medical Sciences for their invaluable assistance in editing this article.

\section{References}

Bezerra, E. S., Simão, R., Fleck, S. J., Paz, G., Maia, M., Costa, P. B., . . Serrão, J. C. (2013). Electromyographic activity of lower body muscles during the deadlift and still-legged deadlift. Journal of Exercise Physiology Online, 16(3), 30-39.

\section{Eleanor (2010). Cram's introduction to surface electromyography: Jones \& Bartlett Publishers.}

Chang, Y.-W., Hung, W., Wu, H.-W., Chiu, Y.-C., \& Hsu, H.-C. (2010). Measurements of foot arch in standing, level walking, vertical jump and sprint start. International Journal of Sport and Exercise Science, 2(2), 31-38.

D’Lima, D. D., Patil, S., Steklov, N., \& Colwell Jr, C. W. (2011). The 2011 ABJS Nicolas Andry Award:'Lab'-in-aknee: in vivo knee forces, kinematics, and contact analysis. Clinical Orthopaedics and Related Research $®, 469(10)$, 2953-2970.

de Moura, B. M., de Souza Bezerra, E., da Rosa Orssatto, L. B., Sakugawa, R. L., \& Diefenthaeler, F. (2016). Prediction of One Repetition Maximum for 45o Leg Press in Untrained and Trained Elderly Adults.

Escamilla, Zheng, N., Fleisig, G., Lander, J., Barrentine, S., Cutter, G., \& Andrews, J. (1997). The Effects of Technique Variations on Knee Biomechanics During the Squat and Leg Press 887. Medicine \& Science in Sports \& Exercise, 29(5), 156.

Escamilla, R. F. (2001). Knee biomechanics of the dynamic squat exercise. Med Sci Sports Exerc, 33(1), 127-141.

Escamilla, R. F., Fleisig, G. S., Zheng, N., Barrentine, S. W., Wilk, K. E., \& Andrews, J. R. (1998). Biomechanics of the knee during closed kinetic chain and open kinetic chain exercises. Medicine \& Science in Sports \& Exercise(30), 556-569.

Escamilla, R. F., Fleisig, G. S., Zheng, N., Lander, J. E., Barrentine, S. W., Andrews, J. R., . . Moorman, C. T. (2001). Effects of technique variations on knee biomechanics during the squat and leg press. Med Sci Sports Exerc, 33(9), 15521566.

Escamilla, R. F., Francisco, A. C., Kayes, A. V., Speer, K. P., \& Moorman 3rd, C. (2002). An electromyographic analysis of sumo and conventional style deadlifts. Med Sci Sports Exerc, 34(4), 682-688. 
Ferber, R., \& Benson, B. (2011). Changes in multi-segment foot biomechanics with a heat-mouldable semi-custom foot orthotic device.

Franettovich, M. M., Murley, G. S., David, B. S., \& Bird, A. R. (2012). A comparison of augmented low-Dye taping and ankle bracing on lower limb muscle activity during walking in adults with flat-arched foot posture. J Sci Med Sport, 15(1), 8-13. doi:10.1016/j.jsams.2011.05.009

Franklin, S., Grey, M. J., Heneghan, N., Bowen, L., \& Li, F.-X. (2015). Barefoot vs common footwear: a systematic review of the kinematic, kinetic and muscle activity differences during walking. Gait \& posture, 42(3), $230-239$.

Fu, F. Q., Wang, S., Shu, Y., Li, J. S., Popik, S., \& Gu, Y. D. (2016). A Comparative Biomechanical Analysis the Vertical Jump Between Flatfoot and Normal Foot. Paper presented at the Journal of Biomimetics, Biomaterials and Biomedical Engineering.

Gibson, R., \& Habing, T. G. (1994). Leg press: Google Patents.

Keller, T., Weisberger, A., Ray, J., Hasan, S., Shiavi, R., \& Spengler, D. (1996). Relationship between vertical ground reaction force and speed during walking, slow jogging, and running. Clinical Biomechanics, 11(5), 253-259.

Kelly, L. A., Cresswell, A. G., Racinais, S., Whiteley, R., \& Lichtwark, G. (2014). Intrinsic foot muscles have the capacity to control deformation of the longitudinal arch. Journal of The Royal Society Interface, 11(93), 20131188.

Kennedy, P. M., \& Inglis, J. T. (2002). Distribution and behaviour of glabrous cutaneous receptors in the human foot sole. The Journal of physiology, 538(3), 995-1002.

Lack, S., Barton, C., Malliaras, P., Twycross-Lewis, R., Woledge, R., \& Morrissey, D. (2014). The effect of antipronation foot orthoses on hip and knee kinematics and muscle activity during a functional step-up task in healthy individuals: A laboratory study. Clinical Biomechanics, 29(2), 177-182.

Larson, M. A. (2013). Comparison of EMG Latencies of the Tibialis Anterior and Soleus in Barefoot and Shod Conditions During Walking.

Letafatkar, A., Zandi, S., Khodayi, M., \& Vashmesara, J. B. (2013). Flat Foot Deformity, Q Angle and Knee Pain are Interrelated in Wrestlers.

Lloyd, D. G., \& Besier, T. F. (2003). An EMG-driven musculoskeletal model to estimate muscle forces and knee joint moments in vivo. Journal of Biomechanics, 36(6), 765-776.

Murley, G. S., \& Bird, A. R. (2006). The effect of three levels of foot orthotic wedging on the surface electromyographic activity of selected lower limb muscles during gait. Clinical Biomechanics, 21(10), 1074-1080.

Murley, G. S., Menz, H. B., \& Landorf, K. B. (2009). Foot posture influences the electromyographic activity of selected lower limb muscles during gait. J Foot Ankle Res, 2(1), 35-35.

Murphy, D., Connolly, D., \& Beynnon, B. (2003). Risk factors for lower extremity injury: a review of the literature. British Journal of Sports Medicine, 37(1), 13-29.

Nigg, B. M. (2001). The role of impact forces and foot pronation: a new paradigm. Clinical journal of sport medicine, $11(1), 2-9$.

Qaqish, J., \& McLean, S. (2010a). Foot Type and Tibialis Anterior Muscle Activity during Stance Phase of Gait. International Journal of Physiotherapy \& Rehabilitation, 1(1), 19-29.

Qaqish, J., \& McLean, S. (2010b). Foot type and tibialis anterior muscle activity during the stance phase of gait: A pilot study. . International Journal of Physiotherapy \& Rehabilitation, 1(1), 19-29.

Ritchie, C., Paterson, K., Bryant, A. L., Bartold, S., \& Clark, R. A. (2011). The effects of enhanced plantar sensory feedback and foot orthoses on midfoot kinematics and lower leg neuromuscular activation. Gait \& posture, 33(4), 576581.

Sacco, I. C., Akashi, P. M., \& Hennig, E. M. (2010). A comparison of lower limb EMG and ground reaction forces between barefoot and shod gait in participants with diabetic neuropathic and healthy controls. BMC musculoskeletal disorders, 11(1), 24.

Sporndly-Nees, S., Dasberg, B., Nielsen, R. O., Boesen, M. I., \& Langberg, H. (2011). The navicular position test - a reliable measure of the navicular bone position during rest and loading. Int J Sports Phys Ther, 6(3), 199-205.

Stevens, J. (2014). Re:"Body mass index categories in observational studies of weight and risk of death" and "Editorial: body mass index and risk of death". American Journal of Epidemiology, kwu299.

Tenforde, A. S., Yin, A., \& Hunt, K. J. (2016). Foot and ankle injuries in runners. Physical medicine and rehabilitation clinics of North America, 27(1), 121-137.

Tomaro, J., \& Burdett, R. C. (1993). The effects of foot orthotics on the EMG activity of selected leg muscles during gait. journal of orthopaedic \& sports physical therapy, 18(4), 532-536.

Wawrzyniak, J. R., Tracy, J. E., Catizone, P. V., \& Storrow, R. R. (1996). Effect of closed chain exercise on quadriceps femoris peak torque and functional performance. Journal of athletic training, 31(4), 335. 